\title{
Errata
}

\section{Kordia algicida gen. nov., sp. nov., an algicidal bacterium isolated from red tide}

Jae Hak Sohn, Jung-Hyun Lee, Hana Yi, Jongsik Chun, Kyung Sook Bae, Tae-Young Ahn and Sang-Jin Kim

International Journal of Systematic and Evolutionary Microbiology (2004), 54, part 3, 675-680

The type strain of Kordia algicida is NBRC $100336^{\mathrm{T}}$, not NBRC 1000336 as cited in the paper (Dr Yasuyoshi Nakagawa, NBRC, personal communication).

\section{Dyadobacter hamtensis sp. nov., from Hamta glacier, located in the Himalayas, India}

\author{
P. Chaturvedi, G. S. N. Reddy and S. Shivaji \\ International Journal of Systematic and Evolutionary Microbiology (2005), 55, part 5, \\ 2113-2117
}

The tree shown in Fig. 1 on p. 2114 is incorrect, although the legend to the figure is correct. A corrected version of the tree is shown below.

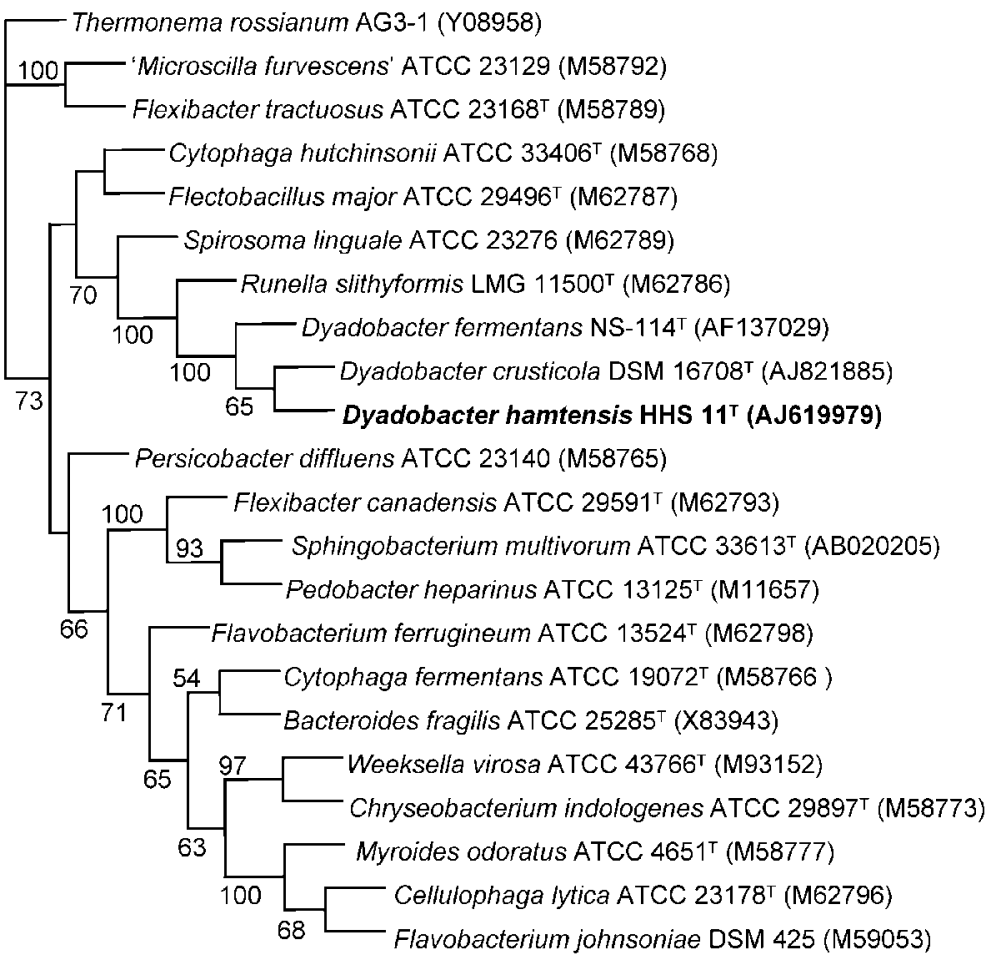

Fig. 1. Neighbour-joining tree, based on 16S rRNA gene sequences (1455 bases), showing the phylogenetic relationship between $D$. hamtensis sp. nov. and species of the genera Dyadobacter, Runella, Spirosoma, Flectobacillus and Cytophaga and other closely related genera. 